\section{Erstes Biologikum zur Therapie des Still-Syndroms zugelassen}

\begin{abstract}
— Die Therapieoptionen bei systemischer juveniler idiopathischer Arthritis ( $\mathrm{s}$ JIA) erweitern sich: Mit Tocilizumab wurde erstmals ein Biologikum für die Therapie der auch als StillSyndrom bezeichneten Erkrankung für Kinder ab zwei Jahren zugelassen. Bei der sJA besteht noch dringender Bedarf für neue Therapien, da die Kinder meist mit erheblichen Nebenwirkungen und insbesondere mit einer ausgeprägten Wachstumsretardierung auf Kortikoide reagieren. Basistherapeutika wie Methotrexat sind kaum wirksam, auch TNF-Hemmer zeigen meist keine adäquate Wirkung.

Die Möglichkeit, die Kinder künftig mit Tocilizumab, einem humanisierten monoklonalen Antikörper gegen den Interleukin-6Rezeptor (IL-6) zu behandeln, ist nach Prof. Dr. Gerd Horneff vom Kinderrheumazentrum St. Augustin daher als therapeutischer Fortschritt zu werten. Der Wirkstoff zeichnet sich durch eine gute klinische Wirksamkeit bei guter Verträglichkeit aus. Er bessert nicht nur
\end{abstract}

die Arthritiden, sondern auch die systemischen Manifestationen der Erkrankung. Dies belegt die TENDER-Studie, in der 112 Kinder drei Monate lang mit anschließender offener Extensionsphase doppelblind, placebokontrolliert zusätzlich zu Kortikoiden mit Tocilizumab behandelt wurden. In der Studie erreichten $85 \%$ der Kinder unter dem IL-6-Hemmer, aber nur 24\% unter Placebo ein ACR-Pedi-30-Ansprechen bei gleichzeitiger Fieberfreiheit (primärer Endpunkt). Der Unterschied betrug $61 \%$, war hochsignifikant und zudem klinisch relevant: „Sehr vielen Kindern wird durch die Behandlung wieder ein weitgehend normales Leben ermöglicht", unterstrich Horneff.

Christine Vetter

Pressegespräch „RoACTEMBRA ${ }^{\oplus}$ - Neuer Standard in der Therapie der SJIA". St. Augustin, 30. Juni 2011.

Veranstalter: Roche Pharma, GrenzachWyhlen und Chugai Pharma Marketing, Frankfurt

\title{
Calprotectin-Bestimmung im Stuhl erleichtert Diagnostik und Monitoring von CED
}

\begin{abstract}
- Calprotectin ist ein kalziumbindendes Protein, das bei Entzündungsprozessen freigesetzt wird. Calprotectin im Stuhl dient als Biomarker für Infektionen sowie für neoplastische Veränderungen im Gastrointestinaltrakt. Das Protein wird sowohl von neutrophilen Granulozyten als auch von Monozyten gebildet. Die biologische Funktion von Calprotectin ist nicht abschließend geklärt. Es wird vermutet, dass ddie Substanz eine Funktion beim Schutz der Zelle vor leukozytären und bakteriellen Proteasen ausübt. Außerdem wird aufgrund der zinkbindenden Eigenschaft eine antibakterielle Wirkung angenommen.
\end{abstract}

Beim Vorliegen einer entzündlichen intestinalen Erkrankung wandern Granulozyten ins Darmlumen und setzen Calprotectin frei, das mit dem Stuhl ausgeschieden wird. Die Konzentration im Stuhl korreliert mit der Anzahl der neutrophilen Granulozyten, die in das Darmlumen migrieren und hier Calprotectin freisetzen. Die Konzentration zeigt das Aus- maß eines entzündlichen Geschehens im Darm an.

Zahlreiche Publikationen konnten belegen, dass der Nachweis von Calprotectin im Stuhl sehr gut mit den histologischen und endoskopischen Befunden der Krankheitsaktivität bei Patienten mit chronisch-entzündlichen Darmerkrankungen (CED) korreliert. Deshalb dient die Bestimmung von Calprotectin im Stuhl auch dazu, den Therapieerfolg bei diesen Erkrankungen objektiv zu dokumentieren sowie durch die Überwachung von Patienten im symptomfreien Intervall ein Rezidiv möglichst frühzeitig festzustellen.

Die R-Biopharm AG bietet mit RIDASCREEN ${ }^{\circledR}$ Calprotectin und weiteren ELISA und Schnelltesten hervorragende Produkte für eine umfangreiche und verbesserte Diagnostik in der Gastroenterologie.

Nach Informationen von R-Biopharm AG, Darmstadt

\section{0-jährige Erfolgsgeschichte}

Als Wegbereiter der orthomolekularen Ernährungsmedizin in Deutschland schaut Orthomol in diesem Jahr auf eine Erfolgsgeschichte von 20 Jahren zurück. Im Jahr 1991 mit zunächst nur zwei Mitarbeitern gegründet, machte das Familienunternehmen die Produktkategorie „ergänzende bilanzierte Diät" in Deutschland bekannt. Als erstes und auch heute noch führendes Unternehmen in Deutschland entwickelt und vertreibt Orthomol ausgewogen dosierte und indikativ zusammengesetzte Mikronährstoffkombinationen. Mittlerweile sind in der Produktpalette über 20 indikationsspezifische orthomolekulare Produkte, die weltweit in 19 Ländern erhältlich sind - Tendenz steigend.

Nach Informationen von Orthomol Langenfeld

\section{Kombinierte Therapie}

Ein wichtiges Therapieziel bei Rhinosinusitis ist neben der Sekretolyse die Eindämmung der überschießenden Entzündungsreaktion. Sinupret ${ }^{\oplus}$ besitzt eine breit angelegte antiinflammatorische Wirkung über die Inhibition verschiedener Entzündungsmediatoren des Immunsystems. Neue Daten zeigen, dass das Phytotherapeutikum beispielsweise die Enzyme Cyclooxygenase-2 und 5-Lipoxygenase hemmt. Beides sind wichtige Faktoren bei der Produktion von Prostaglandinen bzw. Leukotrienen. Sinupret ${ }^{\oplus}$ hemmt die Immunreaktion nicht vollständig, sondern dämmt lediglich die überschießende Entzündungsreaktion ein.

Die antiinflammatorische Wirkweise ergänzt die sekretolytische Wirkung von Sinupret ${ }^{\oplus}$. Beides zusammen unterstützt die Wiederherstellung vonVentilation und Drainage und die mukoziliäre Clearance kann das zähe Sekret mitsamt Pathogenen wieder abtransportieren.

Für die Wirksamkeit von Sinupret ${ }^{\oplus}$ sind vor allem Flavonoide verantwortlich. Sie sind die häufigsten sekundären Pflanzeninhaltsstoffe und kommen in vielen Früchten, aber auch in Gemüsesorten oder Rotwein vor. Durch seine antiviralen und antibakteriellen Eigenschaften wirkt Sinupret ${ }^{\circledR}$ nicht nur den äußerst unangenehmen Symptomen der Rhinosinusitis entgegen, sondern bekämpft gleichzeitig auch die Ursachen der Erkrankung.

Nach Informationen von Bionorica, Neumarkt 